\title{
Current Aspects in Diagnostics and Treatment of Urogenital Chlamydia in Men
}

\author{
Mugutdinova A.S. ${ }^{1, *}$ Magomedova A.M. ${ }^{1}$ Khatsieva M.S. ${ }^{2}$ \\ ${ }^{1}$ Dagestan State Medical Academy, Makhachkala, Russia \\ ${ }^{2}$ Chechen State University, Grozny, Russia \\ *Corresponding author. Email: amina2706@yandex.ru
}

\begin{abstract}
The paper discusses the possibilities of treating chronic chlamydia prostatitis. The retrospective analysis of literary sources made it possible to conclude that urogenital chlamydia is one of the diseases, which treatment is most difficult due to the fact that many of its manifestations are asymptomatic and it is necessary to diagnose it later when the disease goes into a chronic state. The literature analysis made it possible to suggest that despite numerous studies in this area, the opinions of scientists on this matter vary and there is still no unambiguous view on the role of chlamydia infection in the development of prostatitis, which is largely caused by difficulties in identifying the causative agent in prostate tissue. Although all studies based on the principles of evidence-based medicine confirm that urogenital chlamydia infection can cause urethritis, epididymitis and male infertility. This study also considers the development of a new comprehensive method for diagnostics and treatment of chronic chlamydia prostatitis with the assessment of its efficiency based on molecular biology. The experimental group included 50 patients from 18 to 65 years old: 10 patients with chronic prostatitis caused by $\mathrm{C}$. trachomatis as a single-agent infection; 40 patients with chronic prostatitis with combined chlamydial-mycoplasmal and other urogenital infection. The control group included 20 healthy men without chlamydia and other urogenital infections. A comprehensive clinical and laboratory study made it possible to reliably detect its pathogens and establish a niveau diagnosis, and on this basis to prescribe adequate comprehensive therapy. The treatment results demonstrated high efficiency and high tolerability by patients.
\end{abstract}

Keywords: chlamydia, prostatitis, urethritis, PCR, urethroscopy

\section{INTRODUCTION}

Chronic chlamydia prostatitis is the most frequent complication of urinogenital chlamydia characterized by protracted recurrent attacks and difficult treatment $[4,5,9]$. Since Chlamydia trachomatis is one of the most common STD pathogens [1], it is one of the most common causes of the adverse effects of urogenital chlamydia, such as impaired sexual function and infertility, and thus represents an important socially significant problem that causes significant economic and demographic damage to the society $[1,2]$. According to the European guidelines for the treatment of infections caused by Chlamydia trachomatis, up to $90 \%$ of cases of chlamydia infection in women and up to $50 \%$ of cases in men are asymptomatic, which leads to the development of complications of the reproductive system. According to the evidence-based medicine, urogenital chlamydia infection can cause urethritis, epididymitis and male infertility. Clinical manifestations of chlamydia infection depend on the virulence of a causative agent, length of chlamydia stay in the body, topography of damage, severity of local and general reactions of macroorganism. Despite a significant number of studies, there is still no unambiguous opinion on the role of chlamydia infection in the development of prostatitis, which is largely caused by difficulties in identifying the causative agent in prostate tissue [8].

Purpose of the study: The present period is characterized by an extremely high prevalence of sexually transmitted infections (STIs), which, along with the expansion of their spectrum, indicates a serious medical, social and demographic danger of diseases and complications caused by them, one of which is chronic chlamydia prostatitis. Chronic chlamydia prostatitis is the most frequent complication of urogenital chlamydia characterized by protracted recurrent attacks and difficult treatment [5]. Since Chlamydia trachomatis is one of the most common STI pathogens [6] it is one of the most common causes of the adverse effects of urogenital chlamydia, such as impaired sexual function and infertility, and thus represents an important social problem that causes significant economic and demographic damage 
to the society [7]. High frequency of chlamydia mixed infection, as well as the same clinical picture of chronic prostatitis and associated urethritis (often asymptomatic) regardless of the type of microorganisms that caused these processes [5], require the use of a whole range of modern laboratory studies in the examination of such patients [3], as well as the niveau diagnosis, including urinary tract damage [4]. Meanwhile, only PCR or in vitro study that are widely used to diagnose urogenital chlamydia can be false-negative in ascending infection. Thus, the development of new, more effective approaches to the detection of the entire spectrum of bacterial pathogens of chronic chlamydia prostatitis and the niveau diagnosis of the urogenital tract will lead to further optimization of existing approaches to the treatment of this disease and improvement of the system to monitor its efficiency.

\section{METHODS AND MATERIALS}

The study covered 50 patients with chronic chlamydia prostatitis aged from 18 to 65 years (an average of $34 \pm 5.4$ years), with the age of disease from 0.4 to 10 years (an average of $3.4 \pm 0.2$ years). For prostatitis, 23 (46\%) patients were previously unsuccessfully treated. The examination of patients included the collection of a history of the disease with the clarification of the source of infection, sexual partners, data on STI in the past, nature and efficiency of treatment. In order to obtain representative data, all patients were divided into two groups, healthy patients who did not have C. trachomatis and other pathogens of urogenital infections were used as a reference group (control)

Group 1 - patients with chronic prostatitis caused by C. trachomatis as a single-agent infection (10 patients);

Group 2 - patients with chronic prostatitis with a combined chlamydial-mycoplasmal and other urogenital infection (40 patients).

Group 3 - control - 20 healthy men without chlamydia and other urogenital infections.

The clinical examination included the study of subjective symptoms, an assessment of a general objective status, a special study of genitourinary organs thus establishing a clinical diagnosis reflecting the form and nature of the course, niveau diagnosis, complications. The obtained data were entered into an individual patient record, which also noted previously suffered diseases and currently established chronic inflammatory processes.

All patients underwent serological studies to exclude syphilis, HIV, hepatitis B and C. Dry total urethroscopy was carried out to establish a niveau diagnosis by the nature of detected pathological changes in the urethra (including condylomas, papillomas and other urethra neoplasms), assess the efficiency (failure) of therapy, determine the degree of residual effects affecting the recovery of anatomical integrity of urethra. All patients underwent transrectal ultrasound scanning (Transrectal US), which was carried out on Voluson 730 Pro (General Electric Company, Austria) using transverse and longitudinal transrectal echography. The material for laboratory studies of patients and healthy persons of the control group was the urethra discharge (after urinary retention for 4-5 hours). The sampling was carried out after balanus treatment with a sterile cotton ball saturated with isotonic sodium chloride solution. The clinical material for the laboratory study was collected from anterolateral walls of urethra at a depth of $2-3 \mathrm{~cm}$.

\section{RESULTS}

The PCR-based diagnostics made it possible to establish the frequency of detection of a causative agent of urogenital infections in chronic chlamydia prostatitis, as well as the frequency of $\mathrm{Cl}$. trachomatis associations with other STI and opportunistic microorganisms. The chronic prostatitis in each case was diagnosed by ultrasonography (Transrectal US) and palpatory study of a prostate gland with examination of its secretion (varying the number of white blood cells from 15 to 100 in a field of view (x400) of a microscope. The results of total dry urethroscopy, clinical and ultrasonographic examination, in each case chronic chlamydia prostatitis was associated in $2(4 \%)$ cases with recent and in 48 (96\%) - with chronic total urethritis, while in $22(44 \%)$ cases - with chronic unilateral vesiculitis, in $5(10 \%)$ cases with - chronic unilateral epididymitis, and in 22(44\%) cases - with chronic vesiculitis and chronic epididymitis.

As for the association between the severity of endourethral complications of chronic urethritis and the nature of pathogens causing it, the most severe endourethral complications were noted in the association of C. trachomatis and M. genitalium, which occurred in 10 out of 12 cases of transitional and in 5 out of 7 cases of solid urethral infiltrate (in general, this association was observed in $72 \%$ of cases of solid and transitional urethral infiltrate), less often they occurred in chlamydialureaplasmic infection (in 2 out of 12 cases of transitional and in 2 of 7 cases - solid urethral infiltrate). Thus, a set of clinical and laboratory studies revealed that in each of the 50 cases the chronic chlamydia prostatitis in the patients examined was associated with urethritis, in 48 (96\%) cases - with chronic and only $2(4 \%)$ cases with - fresh; in $10(20 \%)$ cases the infectious process was caused by a single-agent infection of $\mathrm{C}$. trachomatis, in $40(80 \%)$ cases - by an association of pathogens, including C. trachomatis, U. urealiticum, M. genitalium, T. vaginalis, $\mathrm{G}$. vaginalis. Moreover, the detection of $\mathrm{C}$. trachomatis and $\mathrm{G}$. vaginalis in 25 cases can indicate not only the key role of such an association in the etiology of chronic prostatitis, but also the need for timely treatment of bacterial vaginosis in female sexual partners of such patients. The obtained results (along with data on immune and interferon status) were taken into account in the development of new approaches to the treatment of chronic chlamydia prostatitis and its concomitant urethral lesions.

Besides, significant violations of immune and interferon status (reduction in interferon- $\alpha$ level in 20 $(50 \%)$ and interferon- $\gamma$ in $40(100 \%)$ patients) in case of 
chronic chlamydia prostatitis indicated the feasibility of using Viferon in its complex therapy (rectally $3 \mathrm{MU}$ once a day for 20 days). Viferon stimulates the proliferation of fibroblasts and increases the production of prostaglandins by them, growth factors and a number of cytokines, including colony stimulating factors, interleukins, interferon. Based on the obtained results of a comprehensive clinical-laboratory study and treatment of 50 patients with chronic chlamydia prostatitis, we proposed some treatment regimens.

Regimen 1 (for the treatment of patients of group 1 with C. trachomatis single-agent infection);

Wilprafen: inside $500 \mathrm{mg} 2$ times a day every $1-1.5$ hours after eating for 21 days;

Viferon rectal suppositories: 3 MU once a day for 20 days;

Wobenzym: inside 5 pills 3 times a day 30 minutes before eating with a large amount of liquid (at least $150 \mathrm{ml}$ ) for 20 days.

The treatment of the $2^{\text {nd }}$ group of patients (chlamydia in combination with other STI) was based on antibiotic therapy, Viferon immunotropic drug, systemic Wobenzim fermentation therapy in combination with other drugs acting on detected pathogens. After 1 month after treatment, complete clinical recovery was noted in 45 $(90 \%)$ cases, eradication of chlamydia and other pathogens of genitourinary infections - in $49(98 \%)$ cases (in one case, the removal of $\mathrm{C}$. trachomatis required retreatment with lymphotropic administration of abactal). According to 2013 clinical recommendations of the Russian Society of Dermatovenerologists and Cosmetologists (RSDC), the duration of the therapy of chlamydia infection of the upper genitourinary system, pelvic organs and other organs depends on the severity of clinical manifestations of the genitourinary inflammatory process, the results of laboratory and clinical studies. Doxycycline monohydrate or jozamycin are the chosen agents. A comprehensive clinical and laboratory study made it possible to reliably detect its pathogens and establish a niveau diagnosis, and on this basis to prescribe adequate comprehensive therapy. The treatment results demonstrated high efficiency and high tolerability by patients.

\section{CONCLUSION}

On the basis of the study, a survey algorithm and the principle of comprehensive therapeutic and preventive measures for chronic chlamydia prostatitis were developed and put into practice. Efficiency and rationality of the developed integrated clinical andlaboratory examination and treatment of patients with chronic chlamydia prostatitis is shown taking into account the identified associations of pathogens. The assessment of diagnostic efficiency, sensitivity, specificity of PCR test systems in "real time" made it possible to recommend the possible use of this method in the study of the etiology of chronic urethrogenic prostatitis. The use of the developed algorithm of clinical laboratory examination and management of patients with chronic urethrogenic prostatitis in practical healthcare makes it possible to optimize the detection of pathogens of the infectiousinflammatory process of the urogenital tract, including in cases of small- or asymptomatic course of infection caused by bacteria and protozoa. In turn, timely etiological diagnostics allows prescribing adequate therapy at various stages of inflammation. The proposed algorithm of clinical-laboratory studies allows choosing the optimal set of therapeutic measures and achieving persistent rehabilitation of a prostate gland and urethra of the patient.

\section{REFERENCES}

[1] Yu.S. Butov, V.Yu. Vasenova, Modern data on immunology of chlamydia infection, Russ. J. of Skin and Sexually Transmitted Diseases 4 (2005) 1-4.

[2] E.E. Bragina, O.E. Orlova, G.A. Dmitriev, Some features of the life cycle of chlamydia, Atypical forms of existence, ZPPP 1 (1998) 3-9.

[3] G.A. Dmitriev, Laboratory diagnostics of bacterial urogenital infections, Medical book, Moscow, 2003, $336 \mathrm{p}$.

[4] I.I. Ilyin, Non-gonococcal urethritis in men, Medicine, Moscow, 2005, 292 p.

[5] V.A. Molochkov, I.I. Ilyin, Chronic urethritis prostatitis, Medicine, Moscow, 1998, 288 p.

[6] V.A. Molochkov, Urogenital chlamydia, Binom, Moscow, 2006, $212 \mathrm{p}$.

[7] V.V. Chebotarev, Debating issues of urogenital infections, Russ. J. of Skin and Sexually Transmitted Diseases 1 (2002) 1-3.

[8] N.I. Chernova, Chlamydial infection in men, Considicum Journal, Moscow, 2014.

[9] B.G. Thomas Taylor-Robinson, Role of Chlamydia trahomatis in genital tract and associated diseases, G. Clin. Pathol. 33 (1980) 205-233.

[10] M.E. Ward, Immunobiology and immunopathology of chlamydia infections, APMIS 103 (1995) 769-795. 